\title{
EXPERIMENTAL TRANSMISSION OF MINOR RESPIRATORY ILL- NESS TO HUMAN VOLUNTEERS BY FILTER-PASSING AGENTS. II. IMMUNITY ON REINOCULATION WITH AGENTS FROM THE TWO TYPES OF MINOR RESPIRATORY ILLNESS AND FROM PRIMARY ATYPICAL PNEUMONIA ${ }^{1}$
}

\author{
By THE COMMISSION ON ACUTE RESPIRATORY DISEASES 2 \\ (From the Respiratory Diseases Commission Laboratory, Regional Hospital, \\ Fort Bragg, N. C.) ${ }^{\mathrm{s}}$
}

(Received for publication March 3, 1947)

\section{INTRODUCTION}

In the previous paper (1) it was shown that minor respiratory illnesses were induced in volunteers by means of bacteria-free filtrates obtained from representative cases of acute respiratory disease. Two distinct types of minor respiratory illness resulted: one, a coryza-like infection with a short incubation period of 1 to 2 days (filtrates $\mathrm{S}-\mathrm{CC}$ and $\mathrm{CC}$ ) ; the other, an infection developing after 5 to 6 days and characterized by prominence of sore throat and minimal nasal symptoms (filtrate ARD). A control group of volunteers given autogenous filtrates remained well. None of the subjects developed primary atypical pneumonia following the inoculation of any of these filtrates.

The present paper reports the results of reinocu-

1 This investigation was supported through the Commission on Acute Respiratory Diseases, Board for the Investigation and Control of Influenza and Other Epidemic Diseases in the Army, Preventive Medicine Service, Office of the Surgeon General, United States Army, and by grants from the Commonwealth Fund, the W. K. Kellogg Foundation, the John and Mary R. Markle Foundation, and the International Health Division of the Rockefeller Foundation, to the Board for the Investigation and Control of Influenza and Other Epidemic Diseases, for the Commission on Acute Respiratory Diseases.

2 Members and professional associates of the Commission on Acute Respiratory Diseases were: John H. Dingle, Lt. Col., M.C., A.U.S., Director; Theodore J. Abernethy, Lt. Col., M.C., A.U.S.; George F. Badger, Major, M.C., A.U.S.; Joseph W. Beard, M.D.; Norman L. Cressy, Major, M.C., A.U.S.; A. E. Feller, M.D.; Irving Gordon, M.D.; Alexander D. Langmuir, Major, M.C., A.U.S.; Charles H. Rammelkamp, Jr., M.D.; Elias Strauss, Major, M.C., A.U.S.

${ }^{8}$ Now located in the Department of Preventive Medicine, School of Medicine, Western Reserve University, Cleveland, Ohio. lation of some of the subjects with agents from the 2 types of minor respiratory illness and from primary atypical pneumonia. These investigations were undertaken in order to determine whether or not immunity followed inoculation with the agents. It was found that homologous immunity was present in individuals who had experienced the "sore throat," long-incubation-period disease (filtrate ARD) ; little or no immunity was demonstrated in those who had had coryza-like illness of short incubation (filtrate S-CC). Cross immunity with either filtrate was not found. Primary atypical pneumonia was induced subsequently in these volunteers who had had prior exposure to ARD and S-CC filtrates.

\section{MATERIALS AND METHODS}

Subjects. The volunteer group consisted of 41 healthy adult white males. Of this number, 16 had participated in the previous study; the remaining 25 were replacements.

Isolation procedures. Isolation was continued without interruption in the group of 16 men who remained for the second half of the study. Isolation precautions for the group of incoming men were instituted as soon as practicable after arrival. Partitions in the hallways on the third floor of the hotel separated 20 of the "new" volunteers into 2 groups of 10 men each. Similar partitions on the second floor divided the remaining 21 into groups of 11 and 10 men, respectively. One of these groups consisted of 6 men previously inoculated and 5 replacements; the other consisted of 10 men who had had previous inoculations.

Inocula. The inocula consisted of the same bacteriafree filtrates ARD, S-CC and CC which were utilized in the first half of the study. In addition, a filtrate of respiratory secretions from a patient with primary atypical pneumonia was employed. The illness in this donor began on May 9, 1945, and was characteristic of a moderately severe infection. Constitutional symptoms, pro- 
TABLE I

Titers of cold hemagglutinins in sera from donor of atypical pneumonia filtrate

\begin{tabular}{r|c|c|c|c}
\hline & \multicolumn{4}{|c}{ Final dilution of serum } \\
\hline $\begin{array}{c}\text { Day of } \\
\text { illness }\end{array}$ & \multicolumn{4}{|c}{} \\
\cline { 2 - 5 } & 8 & 16 & 32 & 64 \\
\hline 2 & $2^{*}$ & \pm & 0 & 0 \\
9 & 4 & 1 & 1 & 0 \\
15 & 2 & 0 & 0 & 0 \\
\hline
\end{tabular}

* $0=$ no visible agglutination.

$\pm=$ doubtful agglutination.

1 to $4=$ increasing degrees of agglutination.

ductive cough and substernal pain were prominent features. Pulmonary infiltration was present in the left and right lower lobes. The febrile course lasted 6 days. There was a slight rise and subsequent fall in the titer of cold hemagglutinins in 3 specimens of sera, examined simultaneously (Table I). Specimens of sputum and pharyngeal washings were collected during the febrile peribd, pooled, and a filtrate prepared. Aerobic and anaerobic cultures of filtrate were sterile.

Plan of study. Four experiments were performed over the course of approximately 7 weeks. Experiment I consisted of the reinoculation of 16 men who had participated in the previous study; 11 men were challenged with ARD or S-CC filtrate and 5 men, formerly inoculated with autogenous washings (Controls), were given filtrate S-CC. Experiment II consisted of the reinoculation of the above 11 men with heterologous filtrates, ARD and $\mathrm{S}-\mathrm{CC}$, respectively, and the inoculation of a control group of 5 "new" volunteers with filtrate ARD. Finally, the 21 men above were given filtrate obtained from a patient with primary atypical pneumonia (Experiment III). Additional studies were made in 2 groups of 10 men each who had formerly received filtrates $\mathrm{CC}$ and $\mathrm{Br}-\mathrm{AP}$ (Experiment IV). These men were inoculated with coryza filtrate $\mathrm{CC}$ in order to test homologous immunity in one and heterologous immunity in the other.

Inoculation procedure. The technique of inoculation and the type of inoculation in general were the same as those employed in the first half of the study. When adequate amounts of filtrate were available the total inoculum was $10 \mathrm{ml}$; this was given to each man in 3 equal doses on a single day. A reduction in total dosage to $6.6 \mathrm{ml}$. was necessary with one filtrate (ARD) and to $5.0 \mathrm{ml}$. in the case of another ( $\mathrm{S}-\mathrm{CC}$ ). These were administered in 2 equal doses on a single day.

Clinical and laboratory studies. Each of the men was observed daily for the development of symptoms and signs of respiratory disease. The procedures employed have been described (1).

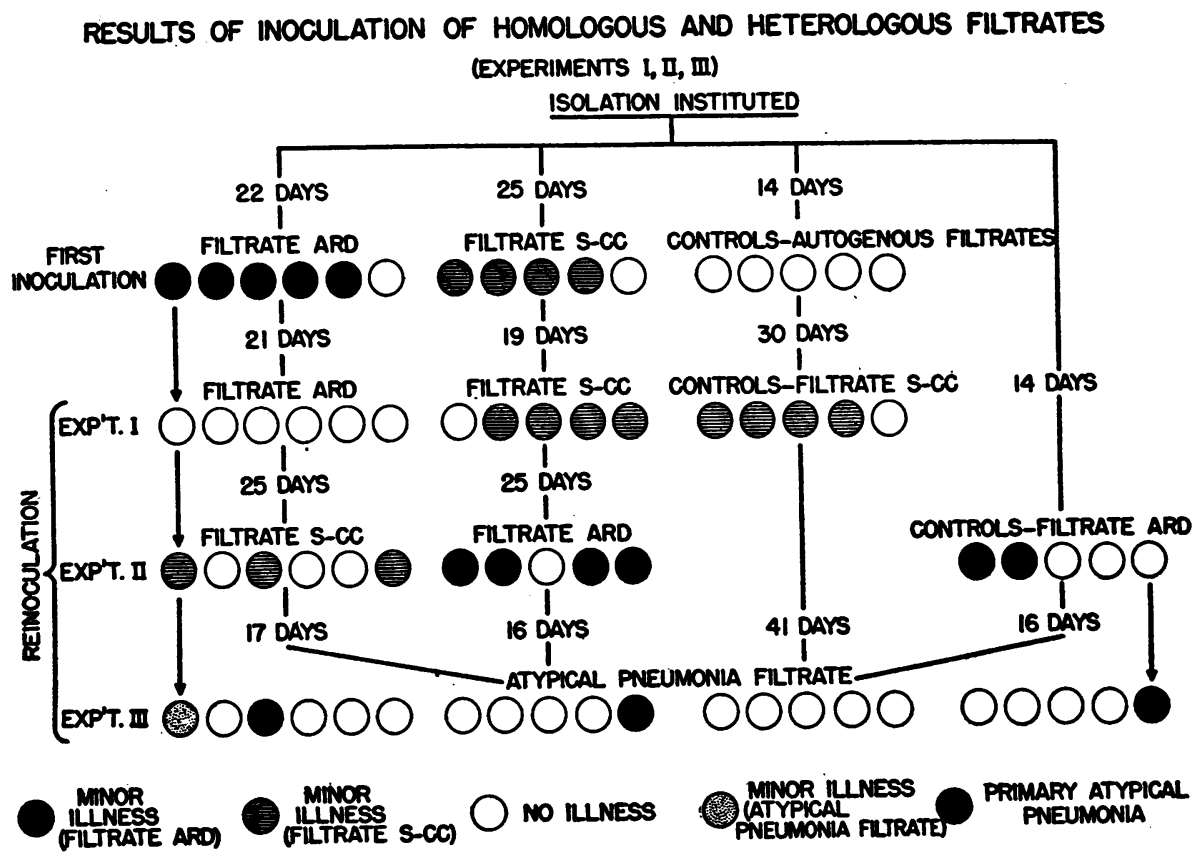

Fig. 1. The Results of Reinoculation of Homologous (Experiment I) and Heterologous (Experiment II) Filtrates and of Challenge with Atypical PneuMonia Filtrate (Experiment III)

Each circle represents a volunteer and the varied shadings indicate the designated type of illness. The sequence of events for each man is indicated by a single vertical column of circles. 


\section{RESULTS}

The effect of inoculation of homologous filtrate (Experiment I)

Approximately 3 weeks after initial inoculation with filtrates ARD and S-CC, 2 groups totaling 11 men, were challenged with homologous filtrates (Figure 1). Five of the 6 men originally exposed to ARD filtrate had experienced illnesses beginning 5 or 6 days after inoculation; 4 of 5 men given $\mathrm{S}-\mathrm{CC}$ inoculum had developed characteristic coryzal illnesses after 1 to 2 days. At the same time a control group of 5 men, who had been inoculated 30 days previously with autogenous washings and had remained well, was given filtrate S-CC.

None of the 6 individuals receiving ARD filtrate a second time developed symptoms or signs of respiratory illness during the course of 25 days. In contrast, 4 of 5 men inoculated with homologous S-CC filtrate developed illnesses. Similarly, 4 of $5 \mathrm{men}$ in the control group receiving S-CC filtrate developed illnesses.

The characteristics of the illnesses in these cases receiving S-CC filtrate were in general similar to those previously noted with this type of inoculum. Onset of symptoms occurred on the 1st or 2nd day in 7 of the 8 cases in both groups. Nasal symptoms and signs were the most characteristic features of the illnesses. In general, however, those who had experienced previous illness as a result of S-CC filtrate suffered from a somewhat milder infection upon reinoculation. This was evidenced principally by the short duration of symptoms which averaged 3 to 4 days.

Comment. The results of this experiment indicated immunity to homologous filtrate in subjects developing the "sore throat," long incubation period disease (ARD). Little or none was demonstrated in those experiencing coryza-like illnesses of short incubation (S-CC). The development of illness in the control group showed that filtrate $\mathrm{S}-\mathrm{CC}$, at least, was infectious in reduced dosage and still active after an additional month's storage. That filtrate ARD was also infectious at this time was indicated by a subsequent experiment, to be described below, in which illnesses were induced in another group of subjects.

\section{The effect of inoculation of heterologous filtrate (Experiment II)}

Twenty-five days after reinoculation of homologous filtrates ARD and S-CC, these 11 men were given heterologous filtrates (Figure 1). Six men, having received 2 inoculations with ARD filtrate, were given $\mathrm{S}-\mathrm{CC}$ filtrate and 5 men, exposed twice to S-CC inoculum, were inoculated with ARD filtrate. A control group of 5 replacements also received ARD filtrate in order to test the activity of this material. The dosages employed were the same as in Experiment I; namely, $6.6 \mathrm{ml}$. of ARD filtrate and $5.0 \mathrm{ml}$. of S-CC filtrate.

As shown in Figure 1, 3 of 6 men given filtrate S-CC and 4 of 5 men inoculated with filtrate ARD developed respiratory illnesses. In the control group 2 of 5 men also became ill.

The types of illness occurring in this experiment were in general the same as those noted previously. The 3 individuals with illness who had been given filtrate S-CC developed onsets of disease on the 2nd and 3rd days after inòculation. Nasal symptoms and signs were prominent in each case. Fever, however, was not present. Those receiving filtrate ARD developed onsets as follows : 2nd day ( 1 case), 5th or 6th day (4 cases), 7 th day (1 case). Symptoms and signs referable to the throat were characteristic. Three individuals displayed fever between the 8th and 10th days, varying between $99^{\circ}$ and $100.8^{\circ} \mathrm{F}$. One subject whose symptoms began on the 2nd day had fever beginning on the 4th day after inoculation. He was moderately ill over the course of 6 days, the maximum temperature reaching $100.8^{\circ} \mathrm{F}$. Constitutional symptoms, sore throat, hoarseness, enlarged tender cervical lymph nodes and signs of pharyngeal inflammation were prominent features of illness.

Comment. The results of this experiment indicated a lack of immunity in subjects given heterologous filtrates ARD and S-CC. There was no indication that antecedent illness, brought about by one filtrate, modified the number or lessened the severity of illness which followed the administration of the heterologous material. Both types of infection, one with a short and the other with a long incubation period, were again induced in subjects even though the inoculum was reduced in amount and all of the individuals had at some 
time previously experienced recent respiratory illness.

The effect of inoculation of filtrate from a case of primary atypical pneumonia (Experiment III)

The 21 volunteers who had participated in Experiments I and II were finally inoculated with filtrate obtained from a single donor early in the course of primary atypical pneumonia.

The experimental group was composed of the following: 6 men who had received filtrate ARD on 2 occasions and then filtrate $\mathrm{S}-\mathrm{CC} ; 5$ men given filtrate S-CC twice and then filtrate ARD; and 2 groups of 5 men, each of whom had received filtrate ARD or S-CC on one occasion. The interval between the last inoculation was 16 or 17 days in 3 of the groups, and 41 days in the case of 1 of the former control groups. The total inoculum administered to each of the 21 men was $10 \mathrm{ml}$. No additional volunteers were available to serve as a control of this inoculum.

Three cases of atypical pneumonia and 1 of minor respiratory illness were observed (Figure 1). The remainder of the group, 17 individuals, failed to show evidence of respiratory infection. Two of the 3 cases of atypical pneumonia and the one instance of minor respiratory illness occurred in individuals who had experienced recent illnesses as a result of inoculation of both filtrates, ARD and S-CC. The remaining case, an individual with atypical pneumonia, had had no infection as a result of previous inoculation with filtrate ARD. No illnesses occurred in this experiment in any of the 5 men originally given autogenous washings and subsequently exposed to filtrate S-CC.

The 3 individuals with pneumonia had illnesses characteristic not only of the naturally acquired infection (2), but also of the experimentally induced disease (3). Onset of symptoms occurred on the 13th, 14th, and 15th days after inoculation, respectively. The illnesses were of mild severity; fever of 5 to 7 days' duration was present in each case. The maximum temperature in 1 individual was $102.4^{\circ} \mathrm{F}$. Unilateral infiltration of one or the other lower lobes was observed in each case. No complications were noted; none of these patients developed cold hemagglutinins in their sera.

The patient with minor respiratory illness first developed symptoms on the 10th day after inocula- tion. His illness began with sore throat and slight nasal discharge. On the 15th day, headache, malaise, coryza, cough and substernal chest pain were noted. Physical examination revealed no significant abnormalities. Pulmonary infiltration was not detected. Significant fever was not present. The duration of symptoms was 9 days. The patient described this illness as a "chest cold" in contrast to the "head cold" he had experienced following the last inoculation (filtrate S-CC).

Comment. The results of this experiment demonstrate that previous minor respiratory illness did not give immunity to primary atypical pneumonia.

These results confirm previous observations that primary atypical pneumonia may be induced in volunteers by means of bacteria-free filtrates (3). In addition, they demonstrate that transmission of the disease may be accomplished with a filtrate obtained from a single donor as well as with the pooled filtrates from several donors. In previous studies, however, it was noted that approximately half of the volunteers developed minor illnesses after inoculation with pooled atypical pneumonia filtrate. Only 1 such illness developed in this experiment.

\section{Additional studies on homologous and heterologous immunity (Experiment IV)}

For further studies on the effect of reinoculation of CC filtrate, 20 additional volunteers were available. One group of $10 \mathrm{men}$ had been inoculated with "coryza" filtrate $\mathrm{CC}$; the other group of similar size had received filtrate $\mathrm{Br}-\mathrm{AP}$ from a patient with respiratory disease termed "bronchitis resembling atypical pneumona" (2). The results of inoculation in the former group had shown 6 coryza-like illnesses with an incubation period of 1 to 4 days. Following the latter inoculation 4 illnesses of ill-defined nature had developed.

Approximately 3 weeks later both groups were reinoculated with $10 \mathrm{ml}$. of "coryza" filtrate CC. Thus, homologous immunity in one group and heterologous immunity in the other, were tested. The results are shown in Figure 2.

Four minor respiratory illnesses developed in men given the heterologous material and 3 in those who were challenged with the same filtrate (CC). Onset of symptoms in these 7 cases occurred on 


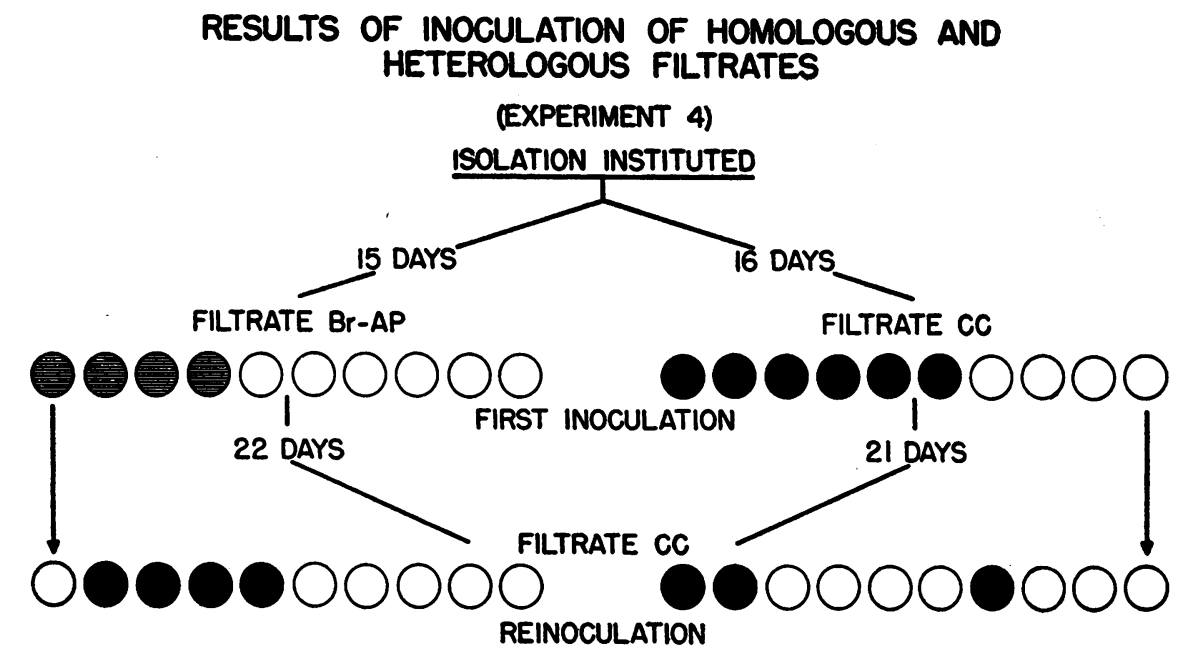

MINOR ILLNESS
(Br-AP FILTRATE)
MINOR ILLNESS
(CC FILTRATE)

Fig. 2. The Results of Reinoculation, with "Common Cold" Filtrate (CC), of 2 Groups Previously Exposed either to the Homologous Filtrate (CC) or to Heterologus Filtrate from a Case Termed "Bronchitis Resembling Atypical PneuMONIA" (Br-AP)

the 1st and 2nd day in 5 cases and on the 9th day in 2 cases. Nasal symptoms and signs of variable intensity were found in all but 1 case. Cough was noted by 3 patients. Other symptoms noted infrequently were headache, malaise, sore throat and chest pain. The illnesses were afebrile and of 2 to 5 days' duration.

Comment. These results confirm the findings in Experiments I and II to a limited extent. Employing 1 type of "coryza" filtrate CC, subjects challenged with the same filtrate again developed characteristic illnesses. Other subjects previously inoculated with filtrate $\mathrm{Br}-\mathrm{AP}$ developed illnesses when exposed to heterologous filtrate CC. Thus, little or no evidence of cross immunity was demonstrated. The explanation for the delayed incubation period of 9 days in 2 cases was not apparent.

Laboratory studies. Examination of the throat flora at 2-day intervals in all subjects failed to show any significant change as result of inoculation in the frequency of the pneumococcus, Beta-hemolytic streptococci, Staphylococcus aureus, $\mathrm{He}$ mophilus influenzae, $H$. hemolyticus, Klebsiella pneumoniae and gram-negative cocci. Furthermore, the distribution of specific types of pneumococci and of Beta-hemolytic streptococci in volunteers, attendants and professional staff revealed no evidence of spread during the course of these experiments.

The total and differential leucocyte counts were within normal limits in all volunteers. No consistent alterations were evident in either type of examination as a result of any of the various types of inoculations. Similarly, the corrected erythrocyte sedimentation rates were normal in all subjects.

A rise in titer of cold hemagglutinins (4-fold increment) was found in the serum of but 1 individual (Table II). This volunteer, during the pre-inoculation control period, showed a cold hemagglutinin titer of 16 . Following the administration of autogenous washings and of filtrate S-CC

TABLE II

Results of cold hemagglutinin tests*

\begin{tabular}{c|c|c|c|c}
\hline \hline \multirow{2}{*}{$\begin{array}{c}\text { Experi- } \\
\text { ment }\end{array}$} & $\begin{array}{c}\text { Number of } \\
\text { observa- } \\
\text { tions }\end{array}$ & \multicolumn{3}{|c}{ Rise in cold hemagglutinin titer } \\
\cline { 2 - 4 } & 4-fold & $<4$-fold & No rise \\
\hline I & 16 & 0 & 1 & 15 \\
II & 16 & 0 & 1 & 15 \\
III & 21 & 1 & 3 & 17 \\
IV & 20 & 0 & 1 & 19 \\
\hline
\end{tabular}

* Based on weekly samples of serum obtained throughout the period of observation. Titer of the last serum specimen before each inoculation was considered the preinoculation titer. 
the maximum titer on repeated occasions was 8. A titer of 32 was noted in the serum 14 days after inoculation of atypical pneumonia filtrate. $\mathrm{He}$ experienced no illness with the latter filtrate.

Appropriate sera from each individual were tested for antibodies against influenza viruses $A$ and B and also streptolysin "O." In no instance was a diagnostic increase in titer observed.

\section{DISCUSSION}

The studies in human volunteers presented in this report and in the preceding paper (1) constituted an attempt to segregate "entities" in the group of unclassified respiratory diseases and to define more clearly the relation between minor respiratory illness and primary atypical pneumonia. These objectives were partially achieved. Two types of minor respiratory illness were induced which appeared to be distinct entities having no demonstrable immunological relationship. The inocula giving these 2 types of minor illness did not induce primary atypical pneumonia, nor did they prevent the subsequent development of primary atypical pneumonia. It would thus appear that 3 separate entities have been dealt with in these experiments. Limitations of the data, however, prohibit the conclusion that the agent of primary atypical pneumonia is not associated with minor respiratory illness. The results will be considered further under the following headings: Minor respiratory illness induced by the ARD filtrate, minor respiratory illness induced by the $\mathrm{CC}$ filtrate, and the relation of minor respiratory illness and primary atypical pneumonia.

Minor respiratory illness induced by the $A R D$ filtrate. Minor respiratory illness, characterized predominantly by sore throat and an incubation period of from 5 to 6 days, was induced with the ARD filtrate. The inoculation of the same filtrate 21 days later demonstrated an apparently solid immunity. This conclusion appears to be valid since the challenge filtrate produced illness in a control group of "new" men inoculated at a later date; and because there seems to be no reason to assume the existence of a refractory state, such as that observed in the ferret by Stuart-Harris and Francis (4), or the operation of the interference phenomenon (5). Moreover, these subjects were sus- ceptible to S-CC filtrate 25 days after they had been challenged with the ARD filtrate (46 days after their initial inoculation).

The minor respiratory illnesses induced by the ARD filtrate, after an incubation of 5 to 6 days, appear to constitute a new entity which has not been described previously in transmission experiments. It seems possible that this entity, despite its mild nature in the recipients (1), is the same as that observed in recruits and termed "undifferentiated acute respiratory disease" (ARD). Clinical studies of these illnesses in recruits have shown them to be primarily noncoryzal respiratory infections (6). The illnesses likewise may be similar to those termed cases of "sore throat" by Van Volkenburgh and Frost (7) in their studies in a civilian population. Epidemiological observations have not defined the incubation period but have suggested the development of immunity as indicated by the absence of epidemics of ARD in seasoned troops as contrasted with recruits (8). Finally, the washings used in these experiments were obtained from a recruit whose illness was considered clinically to be typical of ARD.

Minor respiratory illness induced by CC filtrates. The minor respiratory illnesses induced by filtered washings from 2 cases, referred to here as "common cold," were characterized by predominance of coryza and an incubation period of 1 to 2 days. No appreciable homologous immunity could be demonstrated in the S-CC and CC groups following inoculation with the respective filtrates at intervals of 19 days (S-CC) and 21 days (CC). Heterologous immunity to the ARD filtrate was not demonstrated. The control experiments, in which autogenous filtrates were employed, indicate that these results were not due to mechanical irritation of the respiratory passages or the result of stimulation of a latent agent already present in the respiratory tract.

Minor respiratory illnesses produced by these CC filtrates appear to be similar to, if not identical with, the illnesses transmitted by Kruse (9), Foster (10), Dochez et al (11), and Long et al (12), and termed by them the "common cold." The failure to demonstrate immunity to the CC filtrate in the present experiments is contrary to the opinion generally held that such minor infections have an immunity of approximately 3 or 4 months. They 
are in accord, however, with findings in the Abel Fund Studies (13). Previous investigators did not attempt transmission experiments during the 3 or 4 months following a cold or an experimentally induced infection.

The frequency with which this minor respiratory illness, which resembles the "common cold," occurs in recruits and seasoned troops has not been adequately determined. It is possible, however, that this disease is similar to the minor illnesses observed in field surveys and in dispensaries during the summer and fall months.

Relation of minor respiratory illness and primary atypical pneumonia. Clinical and epidemiological studies of sporadic and epidemic primary atypical pneumonia have shown, in many instances, an apparent association between this disease and minor respiratory illnesses $(2,3,14$ to 17$)$. These findings have led to the hypothesis that the same agent produces both types of infection and that primary atypical pneumonia may actually be an infrequent manifestation of pulmonary involvement in a prevalent minor respiratory disease. Such a theory seems reasonable and is not novel, since it is in accord. with the behavior of such known agents as influenza virus $A$, the meningococcus, etc. This interpretation may likewise be placed on the demonstration, in 1944, that minor respiratory illnesses and primary atypical pneumonia were induced in 50 per cent and 25 per cent, respectively, of a group of volunteers inoculated with pooled filtered secretions of the respiratory tracts of 6 patients with primary atypical pneumonia (3).

The above evidence alone is not sufficient to prove the hypothesis, however, because the presence of only one agent cannot be established. Moreover, minor respiratory illnesses have been infrequent or absent in some outbreaks of primary atypical pneumonia $(18,19)$ and have varied in their relative sporadic frequency $(20)$. It is therefore possible that more than 1 agent may cause both sporadic and epidemic primary atypical pneumonia and minor respiratory illness. In support of this assumption may be the occurrence of cold hemagglutinins in some but not all cases of primary atypical pneumonia (21-24).

In the present study, further evidence was sought in 2 ways, (a) by an attempt to produce primary atypical pneumonia with washings from each of 2 cases (ARD and $\mathrm{Br}-\mathrm{AP}$ ) considered clinically and epidemiologically to be most closely related to that disease, as well as from 2 unrelated cases ( $\mathrm{S}-\mathrm{CC}$ and $\mathrm{CC}$ ) ; and $(b)$ by an attempt to demonstrate the presence or absence of immunity to primary atypical pneumonia in individuals who had previously been infected with ARD and S-CC filtrates.

Primary atypical pneumonia was not induced by the administration of inocula from any of the 4 cases, nor did previous inoculation with the ARD and S-CC filtrates prevent the subsequent development of the disease. Moreover, the incubation periods of the minor respiratory illnesses which did follow the initial inoculations-approximately 5 to 6 days with the ARD filtrate and approximately 1 to 3 days with the S-CC, CC and $\mathrm{Br}-\mathrm{AP}$ filtrates-were much shorter than that found with the AP filtrates; that is, approximately 14 days (3).

It is difficult to interpret the occurrence of only one case of minor respiratory illness following the challenge inoculation of filtrate from a single case of primary atypical pneumonia (Figure 1) because previously uninoculated control subjects were not available. The absence of minor respiratory illnesses was in definite contrast to the results obtained in 1944 when pooled filtrates from 6 donors were employed (3). Theoretical explanations based on the assumption of the presence of 2 agents in the 1944 filtrates, or partial immunity induced by the ARD and S-CC inocula, do not appear to be adequate because of the absence of mild illnesses in the S-CC control group (Figure 1). One possibility appears to be that the agents producing primary atypical pneumonia in the 1944 and 1945 experiments were different. In support of this assumption may be the consistent occurrence of cold hemagglutinins in the experimental cases of primary atypical pneumonia in 1944 and their absence in the 3 cases induced in the present study. Further investigations of this aspect of the problem are needed before a final conclusion can be reached.

\section{SUM MARY}

Experiments were undertaken in order to determine the effects of reinoculation of bacteriafree filtrates obtained from representative donors 
with acute respiratory disease. Homologous and heterologous immunity was tested. In addition, immunity to primary atypical pneumonia was assessed in subjects who were previously exposed to these filtrates and who contracted minor respiratory illness.

Immunity to reinoculation was found in individuals receiving filtrate $A R D$ which induced minor illness of relatively long incubation period (5 to 6 days). Little or none was found in subjects challenged with filtrate $\mathrm{S}-\mathrm{CC}$ inducing a coryza-like illness of short incubation ( 1 to 2 days). Cross-immunity was not demonstrable with either filtràte.

Primary atypical pneumonia developed in 3 volunteers following the inoculation of filtrate from a single donor who had atypical pneumonia. Each of these 3 patients had had 1 or more previous inoculations with ARD or S-CC filtrates; 2 had experienced recent minor respiratory illnesses.

The results of this study provide additional proof that at least 2 filtrable agents, or viruses, may induce minor respiratory illness in man and suggest that these agents are probably distinct from the virus or viruses of primary atypical pneumonia.

\section{ACKNOWLEDGMENTS}

The Commission is indebted to the Administrative Staffs of Selective Service, Camp Operatives Division, and to the Mennonite Central Committee, the Brethren Service Committee and the National Service Board for Religious Objectors for the cooperation which made these studies possible.

The Commission takes pleasure in acknowledging the assistance of Brigadier General H. C. Coburn, Jr., M.C., Colonel R. T. Arnest, M.C., Colonel J. N. Williams, M.C., Lt. Colonel H. C. Brownley, M.C., Lt. Colonel J. M. Kinsman, M.C., Lt. Colonel F. K. Herpel, M.C., Lt. Colonel A. Blumberg, M.C., Captain M. Cohen, M.C., and Captain P. T. Myers, M.C.

The technical staff of the laboratory rendered invaluable assistance: Cpl. Alexander Adler, T/4 A. P. Aan, T/Sgt. L. P. Codifer, S/Sgt. G. M. England, Sgt. D. G. Foltz, T/Sgt. N. Gallenson, T/Sgt. E. Gold, S/Sgt. E. F. Hoffman, S/Sgt. M. H. Kaplan, T/4 G. J. Leuty, Mrs. Dorothy C. Mickle, 1st Lt. W. A. Mickle, Jr., Barbara A. Mulliken, Jessie P. Neeley, 1st Lt. T. J. Oliver, 1st Lt. R. L. Robinson, Irene Salamandra and S/Sgt. W. W. Skatrud. Without the efficient services of 1 st Lt. Clara B. Kwash, A.N.C., 1st Lt. Mary McCoy, A.N.C., and Captain Aaron Ignatow, M.A.C., these investigations would have been considerably restricted. Special thanks are due Mr. Don Jones who acted as Assistant Director of the Civilian Public Service Unit throughout the course of these experiments. Messrs. L. Allen, R. Catlett, A. P. Gault, J. Hostetler, A. K. Landis, K. E. Matthies, M. D. Reimer, M. H. Schroeder, M. Siemens and D. Wenger voluntarily served as attendants.

Finally, the members of the Commission wish to express their appreciation to the volunteers themselves, without whom these experiments would have been impossible.

\section{BIBLIOGRAPHY}

1. Commission on Acute Respiratory Diseases, Experimental transmission of minor respiratory illness to human volunteers by filter-passing agents. I. Demonstration of two types of illness characterized by long and short incubation periods and different clinical features. J. Clin. Invest., 1947, 26, 957.

2. Dingle, J. H., Abernethy, T. J., Badger, G. F., Buddingh, G. J., Feller, A. E., Langmuir, A. D., Ruegsegger, J. M., and Wood, W. B., Jr., Primary atypical pneumonia, etiology unknown. Am. J. Hyg., 1944, 39, 67.

3. Commission on Acute Respiratory Diseases, The transmission of primary atypical pneumonia to human volunteers. I. Experimental methods, II. Results of inoculation, III. Clinical features, IV. Laboratory studies. Bull. Johns Hopkins Hosp., 1946, 79, 97.

4. Stuart-Harris, C. H., and Francis, T., Jr., Studies on the nasal histology of epidemic influenza virus infection in the ferret. II. The resistance of regenerating respiratory epithelium to reinfection and to physicochemical injury. J. Exper. Med., 1938, 68, 803.

5. Rivers, T. M., Immunological and serological phenomena of virus diseases. Lane Medical Lectures: Viruses and Virus Diseases, 1939, Stanford University, California, Stanford University Press.

6. Commission on Acute Respiratory Diseases. Clinical patterns of undifferentiated and other acute respiratory diseases in Army recruits. Unpublished data.

7. Van Volkenburgh, V. A., and Frost, W. H., Acute minor respiratory diseases prevailing in a group of families residing in Baltimore, Maryland, 1928-1930 : prevalence, distribution and clinical description of observed cases. Am. J. Hyg., 1933, 17, 122.

8. Commission on Acute Respiratory Diseases, Acute respiratory diseases among new recruits. Am. J. Pub. Health, 1946, 36, 439.

9. Kruse, W., Die Erreger von Husten und Schnupfen. München. med. Wchnschr., 1914, 61, 1547.

10. Foster, G. B., Jr., The etiology of common colds; the probable role of a filtrable virus as the causative factor: with experiments on the cultivation of a minute micro-organism from the nasal secretion filtrates. J. Infect. Dis., 1917, 21, 451.

11. Dochez, A. R., Shibley, G. S., and Mills, K. C., Studies in the common cold. IV. Experimental transmission of the common cold to anthropoid apes and 
human beings by means of a filtrable agent. J. Exper. Med., 1930, 52, 701.

12. Long, P. H., Doull, J. A., Bourn, J. M., and McComb, F., The etiology of acute upper respiratory infection (common cold). J. Exper. Med., 1931, 53, 447.

13. Reed, L. J., Statistical treatment of the intervals between successive attacks of the common cold. Presented before the American Epidemiological Society, New York, N. Y., April 27, 1934 (Unpublished).

14. Reimann, H. A., and Havens, W. P., An epidemic disease of the respiratory tract. Arch. Int. Med., 1940, 65, 138.

15. Gallagher, J. R., Acute pneumonitis; report of 87 cases among adolescents. Yale J. Biol. and Med., 1941, 13, 663.

16. Iverson, H. A., An epidemic of acute respiratory disease associated with atypical pneumonia. Bull. Johns Hopkins Hosp., 1943, 72, 89.

17. Breslow, L., Epidemic of acute respiratory disease associated with atypical pneumonia. J. Clin. Invest., 1945, 24, 775.

18. Daniels, W. B., Bronchopneumonia of unknown etiology in a girls' school. Am. J. M. Sc., 1942, 203, 263.
19. Young, L. E., Storey, M., and Redmond, A. J., Clinical and epidemiologic features of an outbreak of primary atypical pneumonia of unknown etiology among hospital and medical school personnel. Am. J. M. Sc., 1943, 206, 756.

20. Commission on Acute Respiratory Diseases, Epidemiology of atypical pneumonia and acute respiratory disease at Fort Bragg, North Carolina. Am. J. Pub. Health, 1944, 34, 335.

21. Horstmann, D. M., and Tatlock, H., Cold agglutinins : a diagnostic aid in certain types of primary atypical pneumonia. J. A. M. A., 1943, 122, 369.

22. Commission on Acute Respiratory Diseases, Cold hemagglutinins in primary atypical pneumonia and other respiratory infections. Am. J. M. Sc., 1944, 208, 742.

23. Curnen, E. C., Mirick, G. S., Ziegler, J. E., Jr., Thomas, L., and Horsfall, F. L., Jr., Studies on primary atypical pneumonia. I. Clinical features and results of laboratory investigations. J. Clin. Invest., 1945, 24, 209.

24. Finland, M., Peterson, O. L., Allen, H. E., Samper, B. A., and Barnes, M. W., Cold agglutinins. II. Cold isohemagglutinins in primary atypical pneumonia of unknown etiology with a note on the occurrence of hemolytic anemia in these cases. J. Clin. Invest., 1945, 24, 458. 